\title{
Síndrome de Burnout em profissionais médicos com atividades em uti COVID-19 em
}

\section{Teresina/PI}

\author{
Burnout syndrome in medical professionals with activities in COVID-19 ICU in Teresina/ PI \\ Síndrome de Burnout en profesionales médicos con actividades de UCI COVID-19 en Teresina/PI
}

Recebido: 24/11/2021 | Revisado: 30/11/2021 | Aceito: 02/12/2021 | Publicado: 04/12/2021

\author{
Marcelino José Augusto Cabral \\ ORCID: https://orcid.org/0000-0002-8514-637X \\ Centro Universitário Uninovafapi, Brasil \\ E-mail: marcelinocabral@gmail.com \\ Isaac Vinícius Costa Pimentel \\ ORCID: https://orcid.org/0000-0001-9024-3783 \\ Centro Universitário Uninovafapi, Brasil \\ E-mail: pimentel.isaac@gmail.com \\ Williams Cardec da Silva \\ ORCID: https://orcid.org/0000-0002-7167-4876 \\ Centro Universitário Uninovafapi, Brasil \\ E-mail: willcardec@hotmail.com
}

\begin{abstract}
Resumo
Objetivo: Verificar a prevalência da síndrome de burnout durante a pandemia de COVID-19 entre médicos que trabalharam em UTIs (Unidades de terapia intensiva). Metodologia: Trata-se de um estudo epidemiológico observacional transversal, de abordagem quantitativa, de caráter descritivo, de natureza básica. Os dados utilizados foram adquiridos através de questionário realizado na plataforma de Google Forms. Resultados: houve participação de 42 médicos, sendo 25 homens e 17 mulheres. 85,7\% dos participantes trabalhavam há mais de dois anos. Apenas 12 profissionais realizavam outra profissão. Constatou-se Burnout em 57,1\% dos médicos. Conclusão: é sugestível que ocorreu um número importante de casos positivos da Síndrome de Burnout, sendo necessário atenção ao bemestar mental dos trabalhadores de saúde não apenas durante a pandemia de COVID-19 como em futuras situações de pandemia. Tal cuidado é importante para que se haja um atendimento de qualidade e mais humanitário para os pacientes acolhidos pelos profissionais e para a proteção destes.
\end{abstract}

Palavras-chave: Burnout; Indicativo; COVID-19.

\begin{abstract}
Objective: to verify the prevalence of burnout syndrome during the COVID-19 pandemic among physicians who worked in ICUs (Intensive Care Units). Methodology: This is a cross-sectional observational epidemiological study, with a quantitative approach, descriptive in nature, of a basic nature. The data used were acquired through a questionnaire carried out on the Google Forms platform. Results: 42 doctors participated, 25 men and 17 women. $85.7 \%$ of the participants had been working for more than two years. Only 12 professionals performed another profession. Indicative of Burnout was found in $57.1 \%$ of physicians. Conclusion: it is suggestive that a significant number of positive cases of Burnout Syndrome occurred, requiring attention to the mental well-being of health workers not only during the COVID-19 pandemic, but also in future pandemic situations. Such care is important to provide quality and more humanitarian care for patients cared for by professionals and for their protection.
\end{abstract}

Keywords: Burnout; Indicative; COVID-19.

\section{Resumen}

Objetivo: verificar la prevalencia del síndrome de burnout durante la pandemia de COVID-19 entre médicos que trabajaban en UCI (Unidades de Cuidados Intensivos). Metodología: Se trata de un estudio epidemiológico observacional transversal, con abordaje cuantitativo, de carácter descriptivo, de carácter básico. Los datos utilizados fueron adquiridos a través de un cuestionario realizado en la plataforma Google Forms. Resultados: participaron 42 médicos, 25 hombres y 17 mujeres. El 85,7\% de los participantes llevaba trabajando más de dos años. Solo 12 profesionales realizaron otra profesión. Indicativo de Burnout se encontró en el 57,1\% de los médicos. Conclusión: Es sugestivo que ocurrió un número significativo de casos positivos de Síndrome de Burnout., que requiere atención al bienestar mental de los trabajadores de la salud no solo durante la pandemia de COVID-19 sino también en situaciones pandémicas futuras. Esta atención es importante para brindar una atención de calidad y más humanitaria a los pacientes atendidos por profesionales y para su protección.

Palavras clave: Burnout; Indicativo; COVID-19. 


\section{Introdução}

Derivado do inglês, o termo "to burn out" ("queimar-se, consumir-se" em português) caracteriza a Síndrome de Burnout (SB), a qual foi citada pela primeira vez, em 1974, pelo psicanalista Herbert Freudenberger ao observar uma perda de prazer em relação ao seu trabalho, relacionando a sensação de esgotamento à falta de estímulo decorrente da escassez de energia emocional. Além desses sintomas, o psicanalista incluiu fadiga, depressão e irritação como pertencentes à sintomatologia. Sendo assim, considerada consequência do estresse ocupacional duradouro. (Moreira et al. 2018)

A primeira pesquisa científica a respeito de Burnout foi realizada em 1970 pelo psicanalista Herbert Freundenberger. Este desenvolveu uma pesquisa em pessoas jovens que davam apoio à usuários de substância psicoativas, os quais sentiam falta de motivação e prestavam assistência desumana devido às dificuldades de aceitação ao tratamento por parte dos enfermos. Neste período a síndrome teve o conceito como exaustão emocional e física relacionada ao trabalho. Para se obter uma compreensão mais favorável no decorrer dos anos após algumas mudanças, acredita-se que está diretamente relacionada às questões interpessoais, nas funções do trabalhador e na qualidade de vida. (Lopes et al. 2016)

Burnout é uma consequência gerada através do estresse crônico no ambiente de trabalho. Na definição teórica se tem quatro percepções baseadas na etiologia: ambulatorial, sociopsicológica, associativo e sócio habitacional. A mais aplicada é sociopsicologia. (Santos et al. 2017)

Sem embargo, em 1999, a psicóloga Christina Malasch e o psicólogo Michael Leiter finalmente definiram a SB, uma síndrome composta pelos tripés exaustão emocional, despersonalização e falta de realização profissional. Assim, o emocional esgotado se relaciona a cansaço, insatisfação do profissional de saúde e diminuição da eficiência no cuidado ao paciente; a despersonalização, caracteriza-se por uma baixa empatia profissional e pelo distanciamento voluntário em relação aos pacientes e aos colegas profissionais, a despeito de situações infectocontagiosas de exceção como a pandemia viral por Covid19; a desvalia profissional associa-se ao sentimento de incapacidade produtiva, de autoestima reduzida em relação ao trabalho. (Prado, 2016)

Segundo Giovanne (2017), Burnout pode se iniciar ainda na graduação e progredir durante a vida profissional. Sendo assim, é necessário que no diagnóstico seja realizado precocemente, para que a intervenção seja executada o mais cedo possível, evitando repercussões sintomatológicas da Sìndrome de Burnout.

Lima et al. citam que a SB decorre da presença de estresse emocional contínuo, sobretudo em virtude do constante cuidar de pessoas e da responsabilidade do profissional médico (Lima et al., 2013). Deste modo, diante do cenário pandêmico, o risco aumentado de SB decorre da pressão cada vez maior para a incorporação de condutas e de procedimentos, na ausência inicial de protocolos com segurança e eficácia científica comprovada para o tratamento dos pacientes portadores de Covid19. Assim, visando resultados imediatos em virtude do aspecto inseguro e muitas vezes súbito dessa infecção viral, o que, em contrapartida, se opõe à conjuntura de mortes em massa na qual os profissionais são obrigados a assistir diariamente.

O estresse ocupacional acomete 70\% dos trabalhadores brasileiros e desses 30\% sofrem da Síndrome de Burnout, interferindo de forma significativa na população, gerando um problema de saúde pública devido à alta incidência desta doença ocupacional. Diante das circunstâncias, a síndrome de Burnout é a razão para quais muitos pesquisadores iniciaram estudos com profissionais, especialmente da área de saúde, por ter relação com situações estressores que podem apresentar as dimensões da síndrome que são a exaustão emocional, a descrença e a eficácia profissional. (Proenci et al. 2017)

Os profissionais médicos submetidos a trabalho em turnos e à jornada extenuante diária de combate ao vírus, apresentam tendência significativa à SB (Ladou et al.,2016). Essa vulnerabilidade aumentada decorre da interação dos profissionais médicos com uma carga significativa de responsabilidade, de proteção e de cuidado com os seus pacientes. (Tironi et al., 2016) 
No contexto da pandemia e isolamento social, profissionais de saúde que trabalham na linha de frente no combate a COVID-19 tiveram o surgimento de quadros de estresse ou agravamento destes. A cronicidade dessa condição leva à exaustão física e emocional. (Barba, 2021)

Profissionais de saúde estão sob risco significativo para desenvolver Burnout por exposição a severas mudanças em seu cotidiano. Isso pode gerar impactos psicológicos negativos para as dimensões consideradas pela síndrome de burnout. (Borges, 2021)

Queixas relacionadas ao excesso de dedicação ao trabalho e à falta de tempo com a família tem se mostrado cada vez mais frequentes entre os médicos. Com isso, o tempo de dedicação ao trabalho tem sido apontado como importante fator estressor por esses profissionais. (Barbosa, 2017)

Com isso, este estudo observacional propõe-se a identificar a incidência da Síndrome de Burnout nos profissionais médicos da cidade de Teresina que atuam no atendimento direto dos pacientes portadores de Covid19, bem como pretende ressaltar a importância de iniciar o tratamento sintomatológico da SB. Outrossim, a dificuldade de prevenção e a impossibilidade de eliminar o fator causal evidenciam a necessidade de embasar propostas com dinâmicas específicas sobre a relação 'SB X tratamento dos pacientes com Covid19', buscando o benefício maior de assistência e cuidado integral no combate ao vírus.

\section{Metodologia}

\subsection{Procedimentos Éticos}

A pesquisa foi realizada seguindo as orientações da Resolução 466/2012 do Conselho Nacional de Saúde (CNS), que trata das diretrizes e normas que regulamentam as pesquisas envolvendo seres humanos. Mesmo sem ter contato direto com os profissionais e seus familiares, por se ter utilizado conexão com internet para acesso aos formulários, também foram necessários que os procedimentos éticos da resolução fossem seguidos. Foi respeitado o sigilo quanto aos dados de identificação dos participantes, de modo que só foram transcritos os dados que realmente foram necessários para a pesquisa.

\subsection{Método de Pesquisa}

Trata-se de um estudo epidemiológico observacional transversal, de abordagem quantitativa, de caráter descritivo, de natureza básica, sendo ainda um estudo de levantamento no que se refere aos procedimentos técnicos, e prospectivos por se tratar da análise dos formulários de profissionais envolvidos com o cuidado durante a pandemia do COVID-19.

\subsection{Cenário e Participantes da Pesquisa}

A pesquisa foi realizada com médicos da rede pública e privada de Teresina-PI. Os participantes do estudo foram médicos que participaram da linha de frente no tratamento de pacientes com COVID-19, de modo que foram avaliadas suas percepções durante os anos de 2020 e 2021 acerca do burnout médico durante a pandemia. O período analisado foi compreendido entre março de 2020 e março de 2021.

\subsection{Coleta de Dados}

A coleta de dados foi feita por meio do preenchimento do formulário usando a plataforma do Google Forms, disponibilizado por link digital, onde foram observados e analisados com o preenchimento de um instrumento próprio que se trata de uma ficha de coleta de dados. As variáveis do estudo que foram transcritas abordam dados como: sentimento de esgotamento, exaustão intensa, indisposição para o trabalho, envolvimento com os pacientes, culpa, desânimo, estresse, dentre 
outros fatores avaliados pelo inventário de Maslach durante os meses de março de 2020 a março de 2021, junto com questionário sociodemográfico a fim de verificar quais participantes se encaixam na coleta de dados.

CRITÉRIOS DE INCLUSÃO: Foram incluídos os formulários de médicos que participaram da linha de frente de cuidados durante a pandemia do COVID-19 em Teresina-PI que o preenchimento contemple o instrumento de coleta.

CRITÉRIOS DE EXCLUSÃO: Foram excluídos dos estudos formulários com preenchimento incompletos e que não contemplaram o instrumento de coleta.

\subsection{Organização e Análise dos Dados}

Os dados coletados passaram inicialmente por uma tabulação no software SPSS for Windows, versão 23, para caracterização da amostra do estudo com a utilização de estatísticas descritivas. Posteriormente foi realizada a análise estatística dos mesmos por meio do teste qui-quadrado. O programa R Statistic versão 3.4 .4 foi usado para realização de comparações por meio do teste $\mathrm{Z}$ e teste de Fisher. Os resultados das análises foram organizados em gráficos para facilitar a apresentação dos mesmos.

\section{Resultados e Discussão}

Os resultados foram organizados em três variáveis da Síndrome de Burnout a partir do questionário de Maslach, sendo que para a Exaustão Emocional (Gráfico 1) a pontuação exigida é maior ou igual a 26, para a Despersonalização (Gráfico 2) a pontuação exigida é maior ou igual a 9 e para a Baixa Realização Pessoal (Gráfico 3) a pontuação é menor que 33. Sendo indicativo de Síndrome de Burnout (Gráfico 4) todos os voluntários que se enquadrem nas três variáveis. Durante o período de coleta de dados foram coletadas 42 respostas de profissionais que atuaram na pandemia de covid19 no período compreendido entre março de 2020 a março de 2021.

\subsection{Exaustão Emocional}

Gráfico 1. Exaustão Emocional

\section{EXAUSTÃO EMOCIONAL}

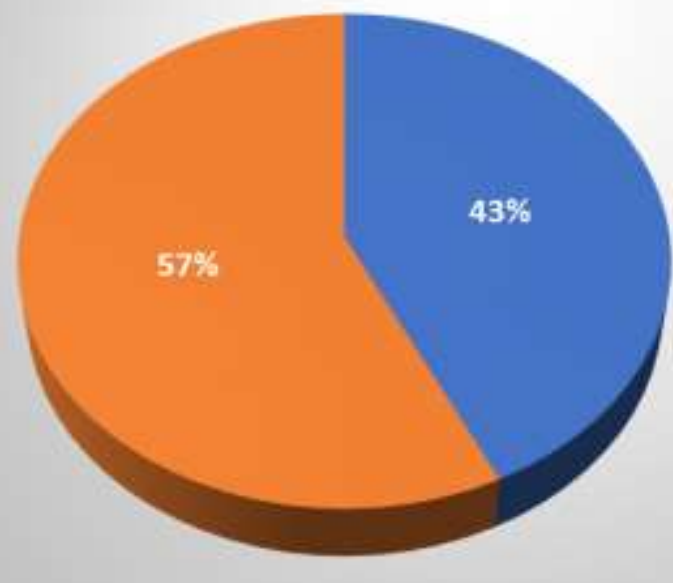

- Näo se enquadra

enquadra

Fonte: Autores. 
No Gráfico 1 apresentamos os resultados da variável exaustão emocional, onde obtivemos um total de 42 respostas, onde 18 (43\%) não se enquadram com exaustão emocional e 24 (57\%) se enquadram.

O aumento do número de casos confirmados e suspeitos de COVID-19, distanciamento da família e amigos, carga exaustiva de trabalho, medo de infecção ou de falecer além do receio de transmitir a outras pessoas, a frustração pelos óbitos dos pacientes são fatores de risco para SB assim como transtornos como ansiedade e depressão. (Borges, 2021)

\subsection{Despersonalização}

Gráfico 2. Despersonalização.

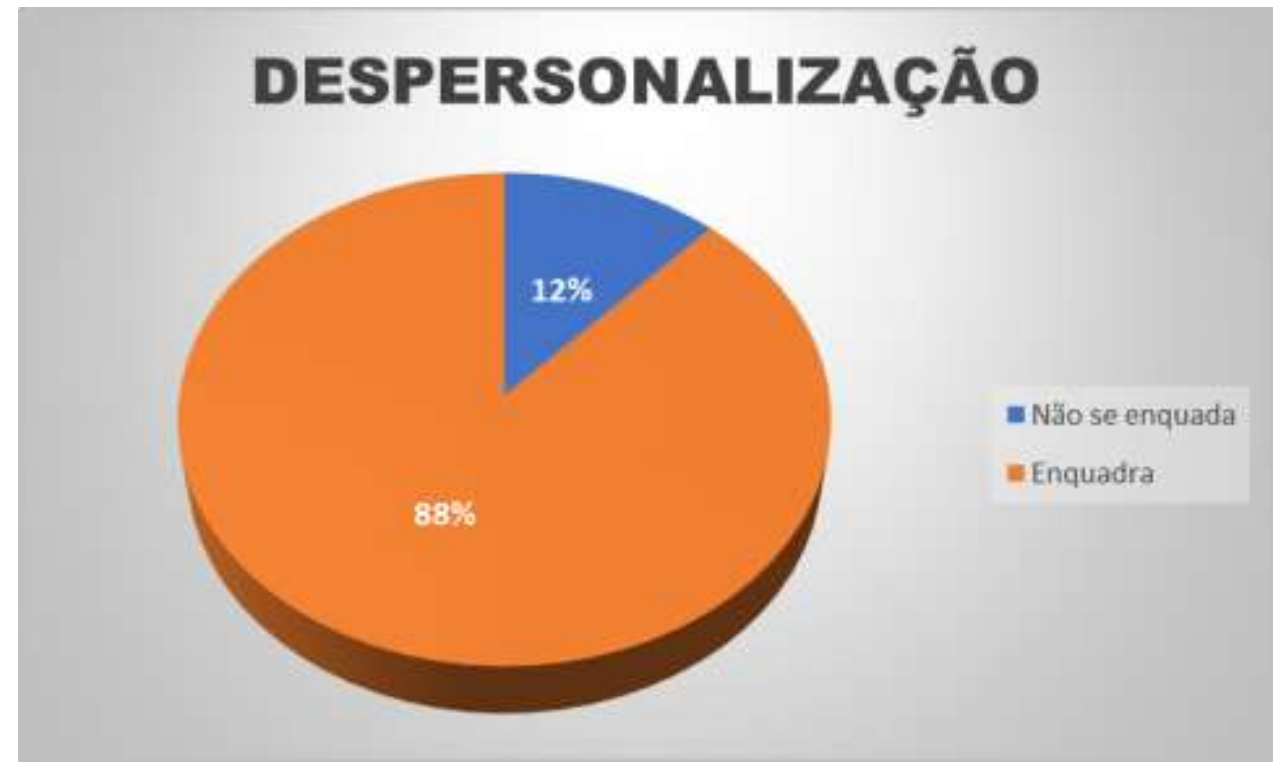

Fonte: Autores.

No Gráfico 2 apresentamos os resultados da variável despersonalização, onde obtivemos um total de 42 respostas, onde 5 (12\%) não se enquadram com despersonalização e 37 (88\%) se enquadram.

\subsection{Baixa Realização Pessoal}




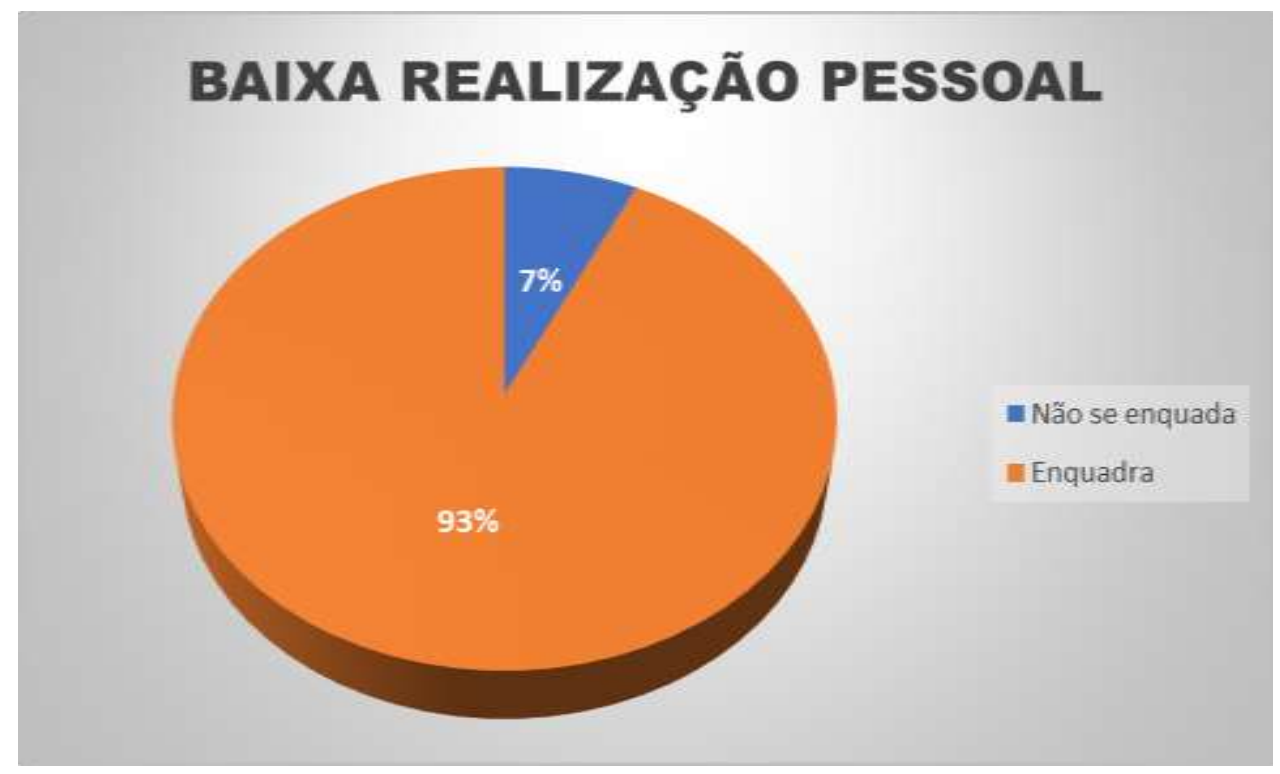

Fonte: Autores.

No Gráfico 3 apresentamos os resultados da variável baixa realização pessoal, onde obtivemos um total de 42 respostas, onde 39 (93\%) se enquadram com em baixa realização pessoal e 3 (7\%) se enquadram.

Baixa eficácia profissional é tendência negativa de autoavaliação no trabalho com relação à profissão. Vivências de insuficiência profissional, esgotamento, fracasso, sentimento de vazio, baixa autoestima e impotência também são frequentes. Somados a esta sintomatologia também podemos encontrar inquietude, dificuldade para concentra-se, irritabilidade, conduta agressiva com companheiros de trabalho e até com familiares. (SAKAE et al. 2017)

\subsection{Síndrome de Burnout}

Gráfico 4. Síndrome de Burnout.

\section{SÍNDROME DE BURNOUT}

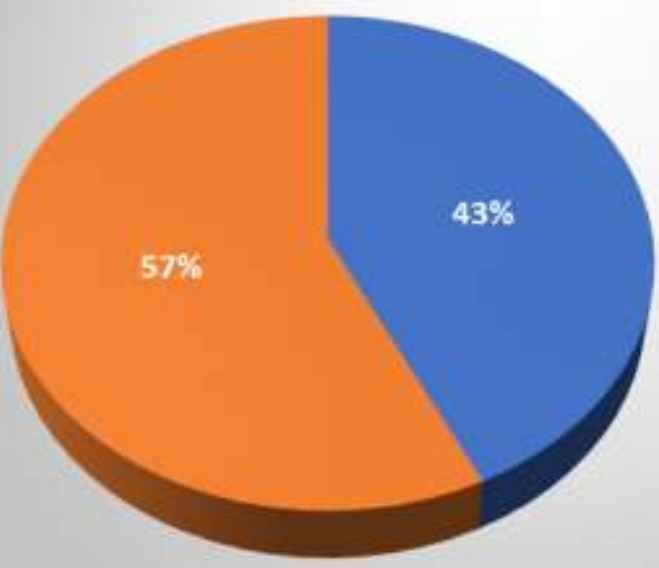

E Não indicativo de Burnnout

EIndicativo de Burnout

Fonte: Autores. 
Ao unir todas as respostas dos participantes da pesquisa que tiveram nas três variáveis apresentadas como resultado a conclusão de que ele se enquadra, chegamos ao resultado de indicativo ou não de SB. Obtivemos um total de 42 respostas, onde 24 (57\%) possuem indicativo de burnout e 18 (43\%) não. Foi observado, então, que 57\% dos voluntários se enquadram com indicativo de Síndrome de Burnout.

Diversos fatores podem ser elencados em relação ao desenvolvimento de burnout, tanto vinculados ao ambiente laboral quanto a estrutura e insumos insuficientes, organização frágil do processo de trabalho e da equipe. Ademais, aspectos inerentes à natureza do ofício, ressaltando aqueles com maior nível de estresse, contato direto com outras pessoas e elevada carga de trabalho são observados na atividade dos profissionais de saúde (Roberti, 2021)

A Síndrome de Burnout que se caracteriza com alterações psicofisiológicas decorrentes do ambiente de trabalho na presença de riscos ergonômicos o que move ao esgotamento físico e mental e leva o profissional a sensações negativas como irritação, medo, angústia, cansaço, ansiedade, desmotivação e desamparo. (Fernandes, 2019)

A Síndrome de Burnout divide-se em três dimensões básicas: exaustão emocional, despersonalização e reduzida realização pessoal no trabalho. (Pereira, 2015)

Maslach e Jackson em 1981 elaboraram a MBI - Maslach Burnout Inventory norteada nas dimensões da exaustão emocional, a despersonalização e a realização profissional, sendo composta por 22 perguntas objetivas relacionadas à frequência com que as pessoas vivenciam determinadas situações em seu ambiente de trabalho. (Pereira, 2015)

A UTI é um ambiente destinado ao tratamento de enfermidades graves, onde os profissionais trabalham em regime de plantão com atendimento individualizado, geralmente invasivo e permeado por emergências. É um ambiente por si só estressante e que requer atenção especial para os profissionais, especialmente em virtude de seu objeto-alvo, que é a saúde dos pacientes, e as graves consequências que podem surgir devido aos efeitos nocivos do estresse crônico. (Barros, 2016)

Devido ao colapso instalado pela pandemia, sabe-se que é difícil controlar a maioria dos fatores de risco para SB. Contudo, intervenções psicológicas como a escuta terapêutica são viáveis e devem ser colocadas em prática. (BARBOSA, 2021)

A síndrome é uma realidade na vida dos profissionais de saúde durante a pandemia da COVID-19. Os estudos avaliados mostram que mais da metade dos profissionais de saúde apresentaram altos níveis de fadiga e exaustão relacionados ao trabalho durante a pandemia, independente do país e condição socioeconômica. (Almeida, 2021)

É importante manter vigilância sobre os fatores que configuram risco para o estabelecimento de SB tais como: inadequadas condições de trabalho, pouco suporte social, baixa remuneração, não reconhecimento pelo trabalho realizado, longa carga horária, contato direto com pessoas em sofrimento físico e psíquico. (Ribeiro, 2020)

\section{Conclusão}

O presente estudo proporcionou o conhecimento de indicativo de Síndrome de Burnout em profissionais de saúde, que estiveram em atividade durante essa pandemia covid19 no período determinado pela equipe de pesquisa. Este trabalho, portanto, vem como ferramenta de gestão em saúde do trabalhador, facilitando a observação de problemas e na busca de resolutividade para essa categoria médica que tanto se dedicou no combate ao novo vírus da covid, destacando-se, ainda, a oportunidade de apresentar um estudo que poderá trazer como benefícios a ampliação dos dados relevantes na literatura acerca do tema, facilitando o embasamento da atuação e de práticas de cuidado com os profissionais de saúde.

Assim, os gestores dos serviços públicos e privados podem utilizar esse estudo para melhorar os serviços e a qualidade do ambiente de trabalho dos profissionais médicos, evitando, assim, a Síndrome de Burnout. Estudos posteriores podem se beneficiar com praticidade de uso de aplicativos para se coletar dados de maneira eficiente e oferecendo segurança ao pesquisador e ao alvo da pesquisa, além de simplificar o processo. Ademais, pode-se buscar os fatores estressores mais 
comuns ou que mais impactam nas dimensões de especificadas de SB. Com mais dados e eficiência nas pesquisas, é possível focar onde se ter mais cuidado no ambiente de trabalho para diminuir os efeitos estressantes nas rotinas de UTI.

\section{Referências}

Almeida, S. L. A. C. et al. (2021). Síndrome de Burnout em profissionais da saúde da linha de frente do COVID-19. Brazilian Journal of Development. 7(7): $66360-66371$.

Barba, M.L. et al. (2021). Síndrome de Burnout na Covid-19: os impactos na saúde dos trabalhadores da saúde. Brazilian Journal of Development. 7(7): 72347-72363.

Barbosa, F. T., Eloi, R. J., Santos, L. M., Leão, B. A., Lima, F. J. C. \& Sousa-Rodrigues, C.F. (2017). Correlação entre a carga horária semanal de trabalho com a síndrome de burnout entre os médicos anestesiologistas de Maceió-AL. Revista Brasileira de Anestesiologia. 67(2): 115-21.

Barbosa, M. V. L. O. et al. (2021). Síndrome de Burnout em profissionais da saúde no contexto da pandemia por COVID-19: revisão integrativa. Brazilian Journal of development. 7(8): 85508-85520.

Barros, M. M. S. et al. (2016). Síndrome de Burnout em médicos intensivistas: estudo em UTIs de Sergipe. Temas em psicologia. 24(1): 377-389.

Borges, F. E. S. et al. (2021). Fatores de risco para a síndrome de burnout em profissionais da saúde durante a pandemia de COVID-19. Revista Enfermagem Atual. 95(33): e-021006.

Borges G. M., Maia J. M., Xavier P. O., Santos A. B. dos R., Barbosa C. C. M., Nogueira V. F. \& Ito A. M. (2021). O impacto da Síndrome de Burnout entre os profissionais de saúde no contexto da pandemia da Covid-19. Revista Eletrônica Acervo Enfermagem. 13. e8375. https://doi.org/10.25248/reaenf.e8375.2021

Fernandes, L. S., Nitsche, T. M. J., Godoy. I. (2017). Síndrome de burnout em profissionais de enfermagem de uma unidade de terapia intensiva. Universidade do Rio de Janeiro. Revista Online de Pesquisa. 9(2): 551-557.

Govêia, C. S., Cruz, T. T. M. D., Miranda, D.B. (2018). Associação entre síndrome de burnout e ansiedade em residentes e anestesiologistas do Distrito Federal. Revista Brasileira de Anestesiologia. 68(5): 442-446.

Ladou, J., Harrison, R. J. (2016). CURRENT Medicina Ocupacional e Ambiental: 5. Ed. Porto Alegre: Artmed.

Lima, R. A., Souza, A. I., Galindo, R. H., Feliciano, K. V. (2013). Vulnerabilidade ao burnout entre médicos de hospital público de Recife. Ciência \& Saúde Coletiva. 18(4): 1051-1058.

Lopes, F. L., Guimarães G. S. (2016). Estudo da síndrome de Burnout em estudantes de psicologia. Psicologia: Ensino \& formação. 7(1): 40-58.

Moreira, H. A., Souza, K. N., Yamaguchi, M. U. (2018) Síndrome de Burnout em médicos: uma revisão sistemática. Revista Brasileira de Saúde Ocupacional. 43(3).

Prado, Claudia. (2016). Estresse Ocupacional: causas e consequências. Revista Brasileira de Medicina do Trabalho. 14(3):285-289

Ribeiro, L. M., Vieira T. de A. \& NakaK. S. (2020). Síndrome de burnout em profissionais de saúde antes e durante a pandemia da COVID-19. Revista Eletrônica Acervo Saúde, 12(11): e5021. https://doi.org/10.25248/reas.e5021.2020

Roberti, B. N. et al. (2021). Síndrome de Burnout em trabalhadores de saúde da linha de frente durante a pandemia do COVID-19 no Brasil. Brazilian Journal of Health Review. 4(5): 21139-21150.

Sakae, T. M. et al. (2017). Prevalência da síndrome de Burnout em funcionários da estratégia da saúde da família em um município no sul do Brasil. Arquivos Catarinenses de Medicina. 46 (1):p. 43-54.

Santos, C. L. C. et al. (2016) Síndrome de burnout em fisioterapeutas: uma revisão sistemática. Revista pesquisa em fisioterapia. 7(1):103-114.

Souza, A. K. S., e Maria, A. L. (2016). Síndrome de burnout em diferentes áreas profissionais e seus efeitos. Revista Acta Brasileira do Movimento Humano. 6(3):1-12. ISSN 2178-2091

Tironi, M. O. S., Teles, J. M. M., Barros, D. S., Vieira, D. F. V. B., Silva, C. M., Martins, D. F. Jr. et al. (2016). Prevalência de síndrome de Burnout em médicos intensivistas de cinco capitais brasileiras. Revista Brasileira de Terapia Intensiva. 28(3): 270-7. 